\title{
Mesure du parcours de protons dans un polymère détecteur
}

\author{
P. MEYER*, M. FROMM*, F. MEMBREY*, A. CHAMBAUDET*
}

A. SERBAT**, J. LAUGIER**

(Manuscrit reçu le 21 décembre 1993)

RÉSUMÉ Le travail présenté dans cet article rentre dans le cadre de la cartographie de dose et de la dosimétrie neutronique utilisant une conversion $(n, p)$, ceci à des fins de radioprotection. Il s'intègre dans une étude visant à la mise au point d'un logiciel permettant, entre autres, à partir de l'observation des paramètres morphologiques d'une trace développée de proton de recul, de déterminer son énergie et son incidence. Ce logiciel permettra d'autre part d'évaluer les paramètres des interactions particule-matière qui ne donnent pas lieu à des traces observables. Dans cette étude, il a été mis en œuvre une analyse mathématique du développement des traces latentes permettant de déterminer expérimentalement la vitesse $V_{\mathrm{T}}$ d'attaque le long de celles-ci à partir de la mesure du parcours des protons. Cette dernière partie fait l'objet de cet article. Celui-ci se limite à la description d'une méthode de détermination du parcours des protons de recul dans un polymère (le CR 39) utilisé comme détecteur solide de traces nucléaires (DSTN). Ces mesures de parcours basées sur une méthode d'analyse mathématique seront comparées à celles déduites du transfert linéique d'énergie (TLE) calculées avec le logiciel TRIM 91. La méthode d'analyse sera vérifiée par des mesures microprofilométriques.

ABSTRACT Within the frame of dose cartography and neutron dosimetry using a neutronproton conversion ( $n, p$ conversion) applied to radiation protection, our final purpose is to develop a computer code making it possible to determine the energy and incidence angle of light ions registered in a CR 39 solid state nuclear track detector (SSNTD) using the morphological parameters of the etched tracks. For this study, a mathematical analysis of the etching process has been developed, allowing determination of the etching velocity $V_{\mathrm{T}}$ along the latent track, by measurement of the particle ranges. This last part constitutes the topic of the present paper which is limited to the description of a method for the measurement of recoil proton ranges in CR 39 SSNTD. The measurement data will be compared with those calculated using linear energy transfer (LET) data given by the TRIM 91 computer code. Experimental results will be treated using microprofilometric measurements.

* Laboratoire de microanalyses nucléaires, UFR Sciences et Techniques, 16, route de Gray, 25030 Besançon Cedex, France.

** ETCA, DPNER-CEB, 16 bis, avenue Prieur de la Côte d'Or, 94114 Arcueil Cedex, France. 


\section{Introduction}

Notre propos est de procéder à une mesure du parcours des protons dans un polymère utilisé comme détecteur solide de traces nucléaires (DSTN) : le CR 39. C'est à l'aide d'une analyse mathématique du développement des traces latentes que nous déterminons la valeur de ceux-ci.

Après avoir succinctement décrit le polymère détecteur, nous exposons dans une première partie la méthode mathématique d'analyse qui conduit à l'obtention de la valeur des parcours, puis nous présentons les expériences et résultats. Dans une seconde partie, nous confirmons la validité de la méthode proposée par une mesure complémentaire : la microprofilométrie. Cette dernière méthode constitue une voie supplémentaire d'obtention de la valeur des parcours. Les résultats obtenus sont commentés, les valeurs expérimentales des parcours sont comparées aux valeurs calculées par le logiciel TRIM 91 [3], puis nous concluons.

\section{Description du DSTN utilisé : le CR 39}

Le CR 39 est un polycarbonate d'allyl diglycol utilisé comme DSTN (Fig. 1) [4] : le passage de particule chargées rapides (dans notre cas des protons d'énergie comprise entre 0 et $2 \mathrm{MeV}$ ) à travers ce polymère a pour effet une dégradation axiale irréversible du matériau à température ambiante. Ces dégâts sont appelés traces latentes, leur diamètre étant de l'ordre de la dizaine d'angströms [14]. Pour être observables et exploitables en microscopie optique, elles doivent être "agrandies". Nous réalisons pour cela une gravure chimique * par une solution de soude concentrée $(7 \mathrm{~N})$ et chaude $\left(70^{\circ} \mathrm{C}\right)$.

Le CR 39 que nous avons utilisé est commercialisé sous le nom de Tastrak $^{\circledR}$. Il est élaboré à l'Université de Bristol par l'équipe du Pr Henshaw $[6,7]$.

\section{Mesure des parcours}

\subsection{Méthode d'analyse}

La méthode d'analyse se réfère à un modèle qui prend en compte deux vitesses de gravure $[2,8,10-13]$ :

- $V_{\mathrm{B}}$ : vitesse d'attaque constante du polymère vierge,

- $V_{\mathrm{T}}$ : vitesse d'attaque longitudinale variable le long de la trace latente.

* L'attaque chimique sera désignée indifféremment par développement chimique ou révélation chimique dans cet article. Notons, cependant, que la traduction exacte du terme "etching" employe habituellement dans la littérature anglo-saxonne est le mot gravure. 
MESURE DU PARCOURS DE PROTONS DANS UN POLYMĖRE DÉTECTEUR

FLUX MONOENERGETIQUE DE PROTONS A INCIDENCE NORMALE

PLAQUE DE C.R 39
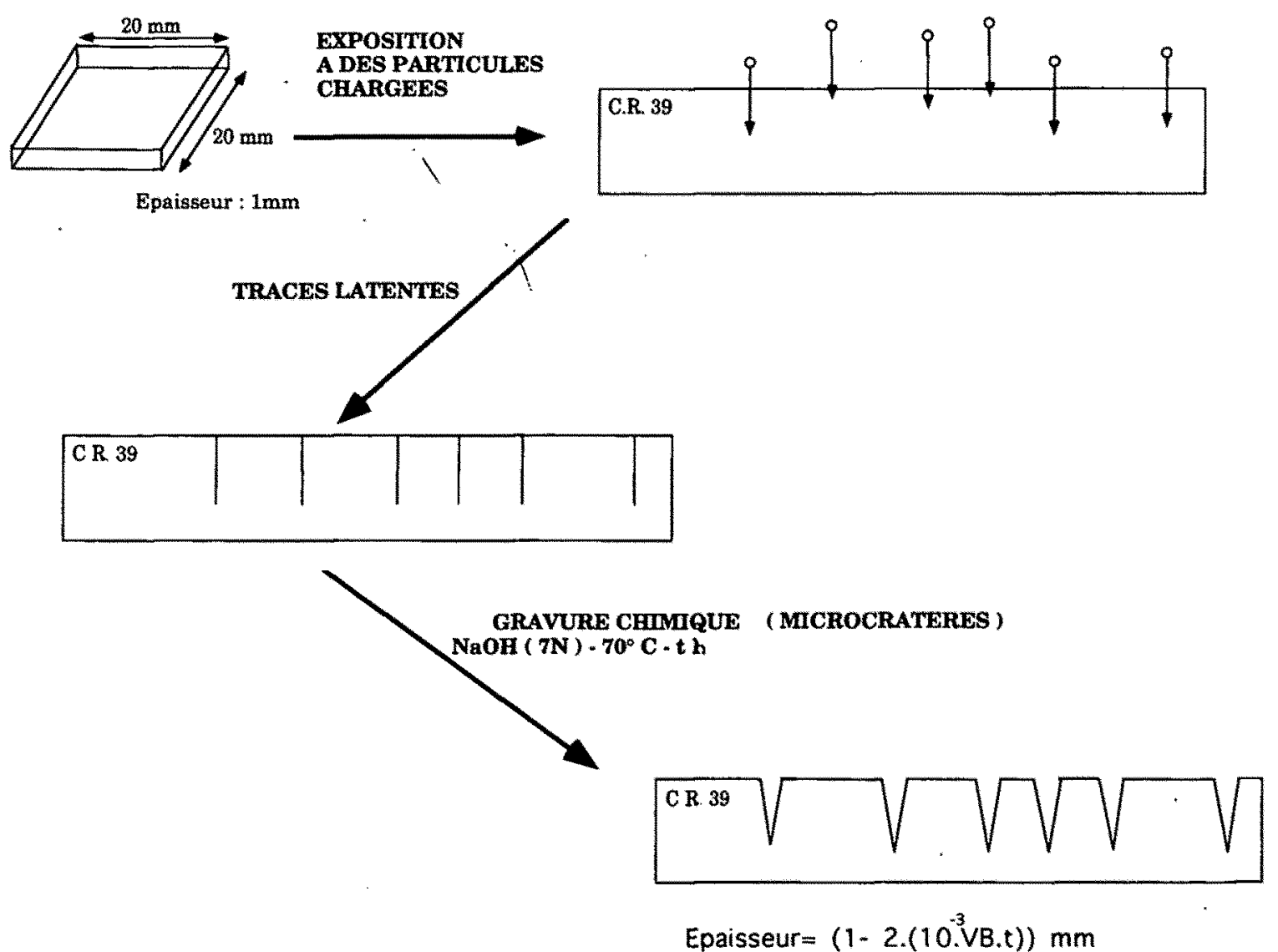

Fig. 1 - Le CR 39 : irradiation et révélation. CR 39 : irradiation and etching.

a) Analyse du développèment d'une trace à incidence normale

Les coordonnées des points $M$ de la paroi de la trace gravée (Fig. 2) dans un plan de coupe sagittale de traces à incidence normale sont [6] :

$$
\begin{gathered}
X=\lambda+\sin (\theta) \cdot V_{\mathrm{B}} \cdot\left[t-\int_{0}^{\lambda} \frac{1}{V_{\mathrm{T}}(x)} \cdot \mathrm{d} x\right] \\
Y=\cos (\theta) \cdot V_{\mathrm{B}} \cdot\left[t-\int_{0}^{\lambda} \frac{1}{V_{\mathrm{T}}(x)} \cdot \mathrm{d} x\right]
\end{gathered}
$$

Lorsque $\lambda$ tend vers $L$, nous obtenons les relations suivantes :

$$
X \rightarrow L+\sin (\theta) \cdot V_{\mathrm{B}} \cdot\left[t-\int_{0}^{L} \frac{1}{V_{\mathrm{T}}(x)} \cdot \mathrm{d} x\right]
$$




$$
Y \rightarrow \cos (\theta) \cdot V_{\mathrm{B}} \cdot\left[t-\int_{0}^{L} \frac{1}{V_{\mathrm{T}}(x)} \cdot \mathrm{d} x\right]
$$

L'intégrale contenue dans ces deux expressions est le temps de développement de la trace révélable noté $t_{L}$ :

$$
\begin{gathered}
X-L \rightarrow V_{\mathrm{B}} \cdot \sin (\theta) \cdot\left[t-t_{L}\right] \\
Y \rightarrow V_{\mathrm{B}} \cdot \cos (\theta) \cdot\left[t-t_{L}\right]
\end{gathered}
$$

Après élévation au carré des deux expressions précédentes, nous obtenons en additionnant :

$$
(X-L)^{2}+Y^{2} \rightarrow\left(V_{\mathrm{B}}\right)^{2} \cdot\left[t-t_{L}\right]^{2}
$$

REPERE :
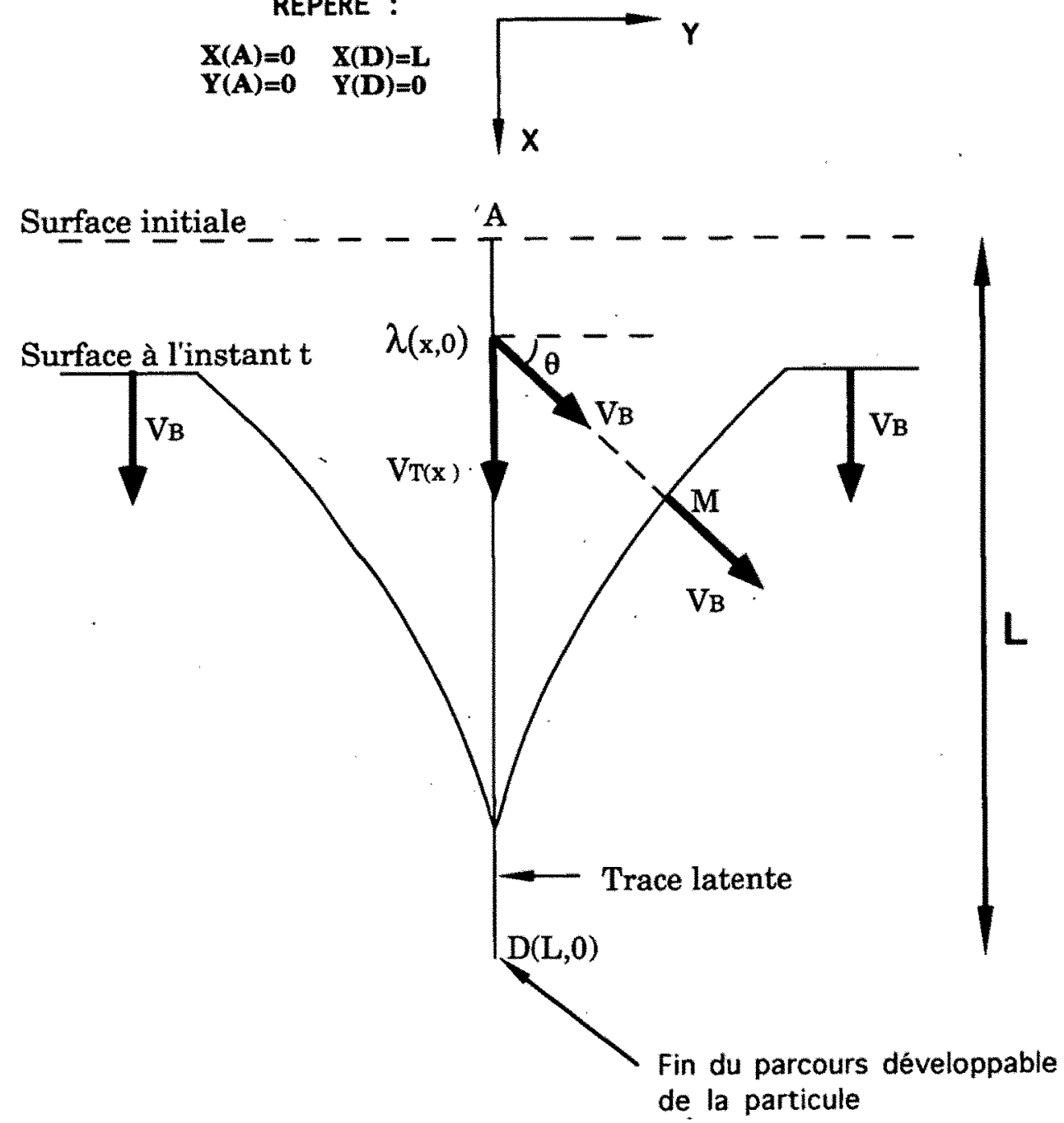

Fig. 2 - Analyse de développement d'une trace à incidence normale. Normal incident track etching analysis. 
Cette relation est l'équation d'un cercle de rayon $V_{\mathrm{B}} \cdot\left(t-t_{L}\right)$ centré en $\mathrm{D}$ extrémité du parcours développable de coordonnées $(L, 0)$. En conséquence, la trace surdéveloppée tend asymptotiquement vers une calotte sphérique de profondeur $P_{C}$ constante alors que le diamètre de son ouverture croît en fonction du temps de surdéveloppement.

\section{b) Principe de la mesure de $L$}

Nous déduisons de la figure 3 la profondeur de la calotte $P_{\mathrm{C}}$ [6] qui est donnée par la relation :

$$
P_{\mathrm{C}}=L-V_{\mathrm{B}} \cdot t_{L}
$$

Le diamètre $\Phi$ s'écrit alors :

$$
\Phi=2 \cdot \overline{\mathrm{BC}}=2 \cdot \sqrt{\overline{\mathrm{DC}}^{2}-\overline{\mathrm{DB}}^{2}}
$$

avec $\overline{\mathrm{DC}}^{2}=\left(V_{\mathrm{B}} \cdot\left(t-t_{L}\right)\right)^{2}$

$$
\overline{\mathrm{DB}}^{2}=\left(V_{\mathrm{B}} \cdot(t-L)\right)^{2}
$$

Nous obtenons en élevant $\Phi$ au carré la relation :

$$
\Phi^{2}=4 \cdot\left\{\left(2 \cdot V_{\mathrm{B}} \cdot t \cdot P_{\mathrm{C}}\right)-\left(V_{\mathrm{B}} \cdot t_{L}+L\right) \cdot P_{\mathrm{C}}\right\}
$$

qui s'écrit comme une forme linéaire du temps $t$ :

$$
\Phi^{2}=\left(8 \cdot P_{\mathrm{C}} \cdot V_{\mathrm{B}}\right) \cdot t-\left(4 \cdot P_{\mathrm{C}} \cdot\left(V_{\mathrm{B}} \cdot t_{L}+L\right)\right)
$$

A condition d'avoir $t>t_{L}$ (surdéveloppement), nous devons observer une croissance linéaire des carrés des diamètres des traces du type :

$$
\Phi^{2}=a \cdot t+b
$$

avec $a=\left(8 \cdot P_{\mathrm{C}} \cdot V_{\mathrm{B}}\right)$

$$
b=-\left(4 \cdot P_{\mathrm{C}} \cdot\left(V_{\mathrm{B}} \cdot t_{L}+L\right)\right)
$$

Le parcours développable $L$ peut être déduit du système suivant :

$$
\left\{\begin{array}{l}
P_{\mathrm{C}}=L-V_{\mathrm{B}} \cdot t_{L} \\
\frac{-b}{4 \cdot P_{\mathrm{C}}}=L+V_{\mathrm{B}} \cdot t_{L}
\end{array}\right.
$$

Donc, en utilisant les expressions de la pente $\alpha$ et de l'ordonné à l'origine $b$, nous pouvons approcher expérimentalement la valeur du parcours révélable $L$ d'une particule à incidence normale ainsi que la profondeur $P_{\mathrm{C}}$ et le temps $t_{L}$, pourvu que sa trace puisse être surdéveloppée :

$$
L=\frac{a}{16 \cdot V_{\mathrm{B}}}-\frac{b}{a} \cdot V_{\mathrm{B}}
$$




$$
\begin{array}{r}
P_{\mathrm{C}}=\frac{a}{8 \cdot V_{\mathrm{B}}} \\
\text { et } t_{L}=\frac{L-P_{\mathrm{C}}}{V_{\mathrm{B}}}
\end{array}
$$
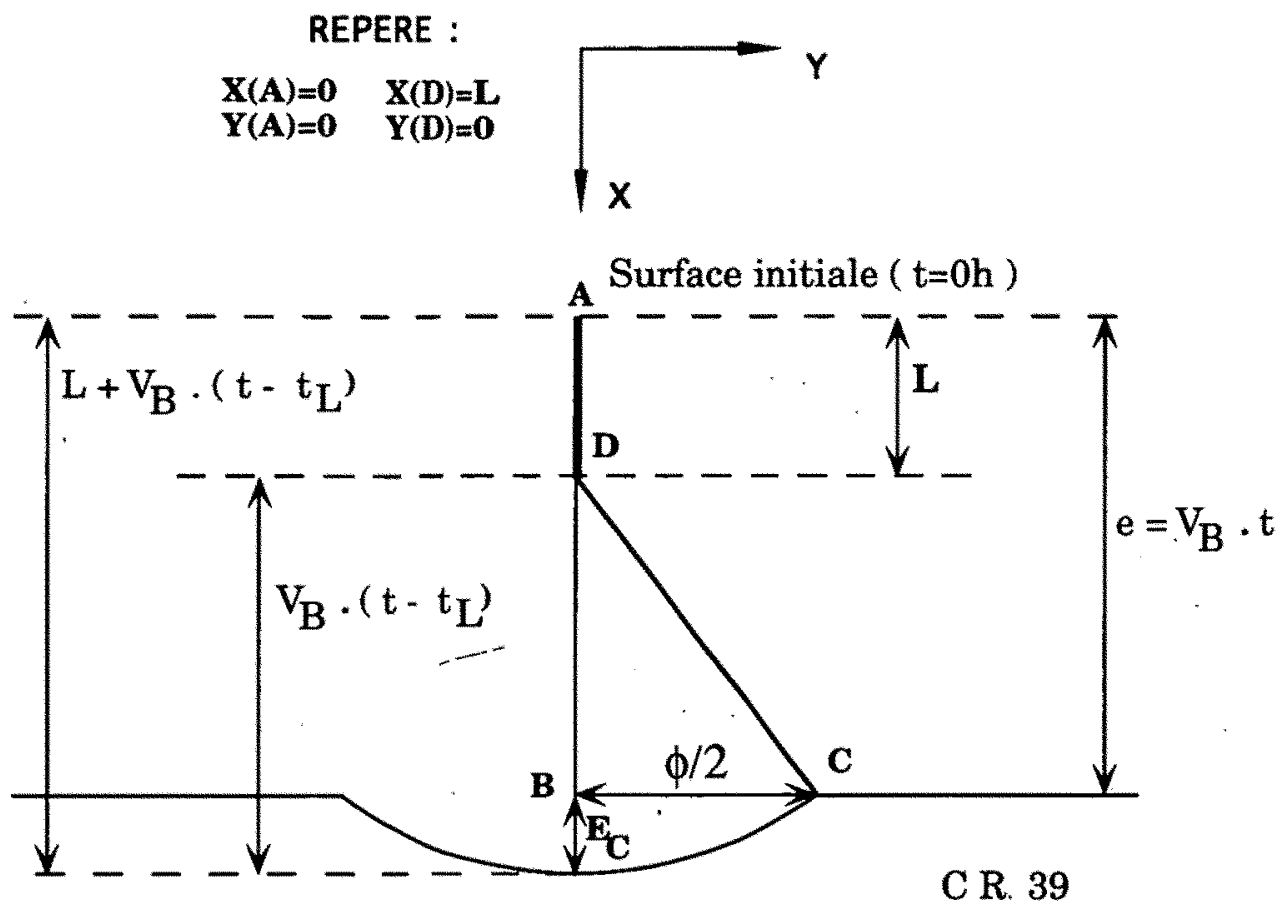

Fig. 3 - Surdéveloppement d'une trace à incidence normale. Overetching of a normal incident track.

\subsection{Expériences et résultats}

\section{a) Irradiations}

Nous avons irradié des détecteurs avec des flux de protons monoénergétiques à incidence normale. Les irradiations ont été réalisées sur l'accélérateur Van de Graaf de l'Etablissement technique central de l'armement (ETCA-CEB-DPNER, Arcueil). Les différentes énergies dont nous disposons sont les suivantes : $238,351,576,1414 \mathrm{keV}$.

\section{b) Développement}

Pour s'assurer une reproductibilité des conditions expérimentales lors du développement chimique, un dispositif a été réalisé (Fig. 4). Celui-ci, régulé en température, assure le transport en circuit fermé de la solution de soude, et permet ainsi de s'affranchir des problèmes d'agitation et de carbonatation du bain durant le développement. Les prélèvements en cours de révélation sont facilités par la présence d'un circuit double : circuit de préchauffage de la 
soude, circuit de développement proprement dit. Il a été vérifié que la concentration de la soude ne variait quasiment pas au cours de développements même très longs (diminution de $1 \%$ de la concentration après $40 \mathrm{~h}$ de développement).

Les échantillons de CR 39 ont été développés pendant des temps $t$ qui étaient très supérieurs à $t_{L}$ : les traces latentes sont donc surdéveloppées. Pour chaque énergie, nous disposions de plusieurs détecteurs qui ont, bien sûr, été développés avec des temps différents.

La gravure chimique a été réalisée dans une solution aqueuse de soude $7 \mathrm{~N}$, à une température de $70^{\circ} \mathrm{C}$. Dans ces conditions expérimentales, $V_{\mathrm{B}}$ est égale à $1,92 \mu \mathrm{m} \mathrm{h}^{-1}$ [9].

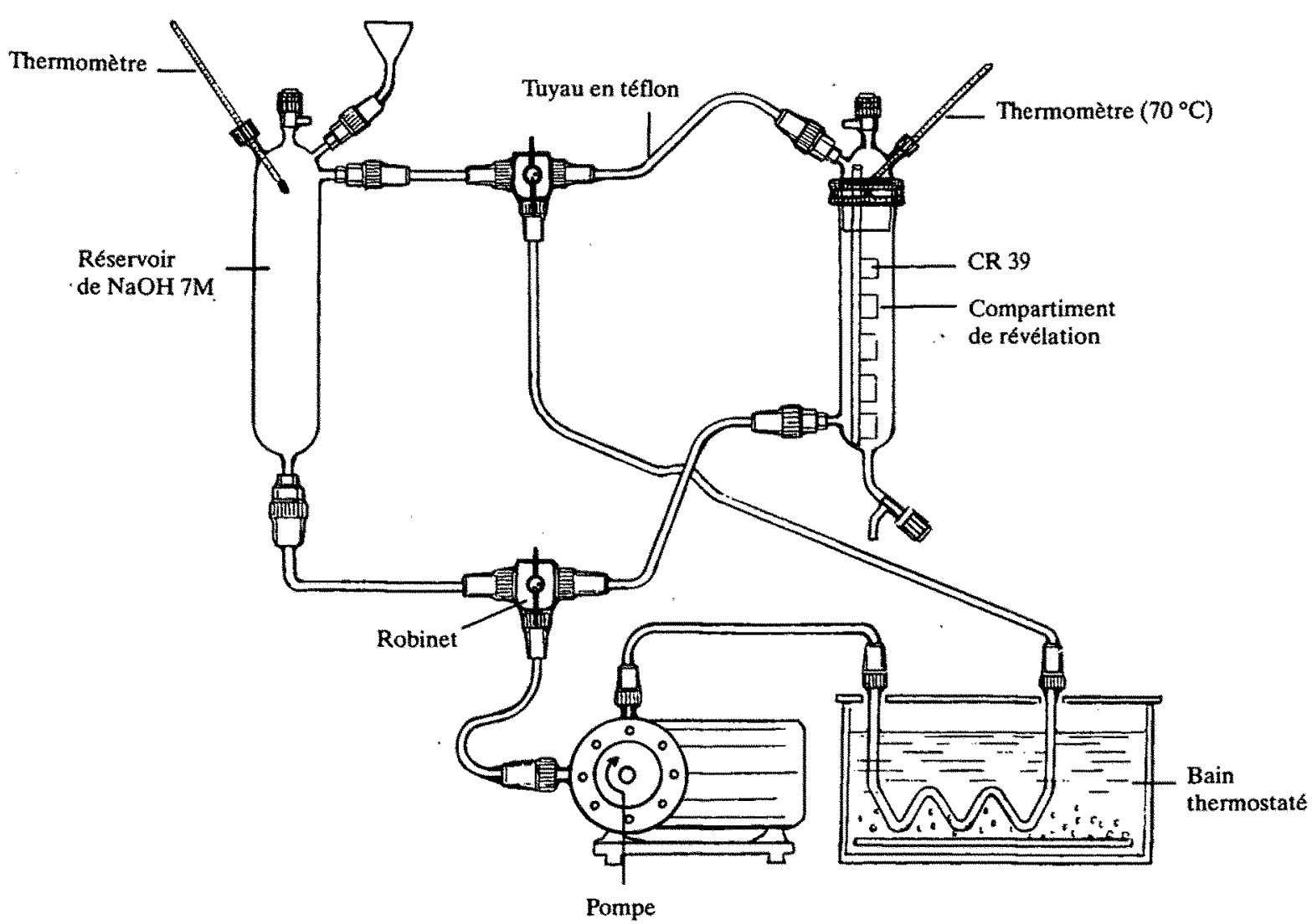

Fig. 4 - Schéma du montage de développement. View of the etching device.

\section{c) Traitement des données expérimentales}

La mesure des diamètres des traces de protons a été effectuée par traitement d'image (système CUE - 2 Image analyser d'Olympus, microscope optique Olympus, grossissement $\times 1500$ ). Nous avons mesuré le diamètre de 50 traces par détecteurs pour avoir une bonne statistique. 
Nous avons représenté sur des graphes (exemple : Fig. 5) les valeurs des diamètres au carré des traces de protons pour chaque énergie en fonction du temps pour $t \gg t_{L}$. On observe bien une croissance linéaire asymptotique du carré des diamètres des traces. Les résultats ont été traités par régression linéaire. Nous avons fait figurer, dans le tableau I, les résultats récapitulatifs des équations des droites et de leurs coefficients de corrélation pour chaque énergie. Les écarts relevés sont imputables à l'expérience :

- difficulté de maintenir la température totalement constante sur des temps très longs (plus de $20 \mathrm{~h}$ ) ;

- résolution du dispositif d'analyses d'images de l'ordre du micromètre ;

- inhomogénéité du CR 39.

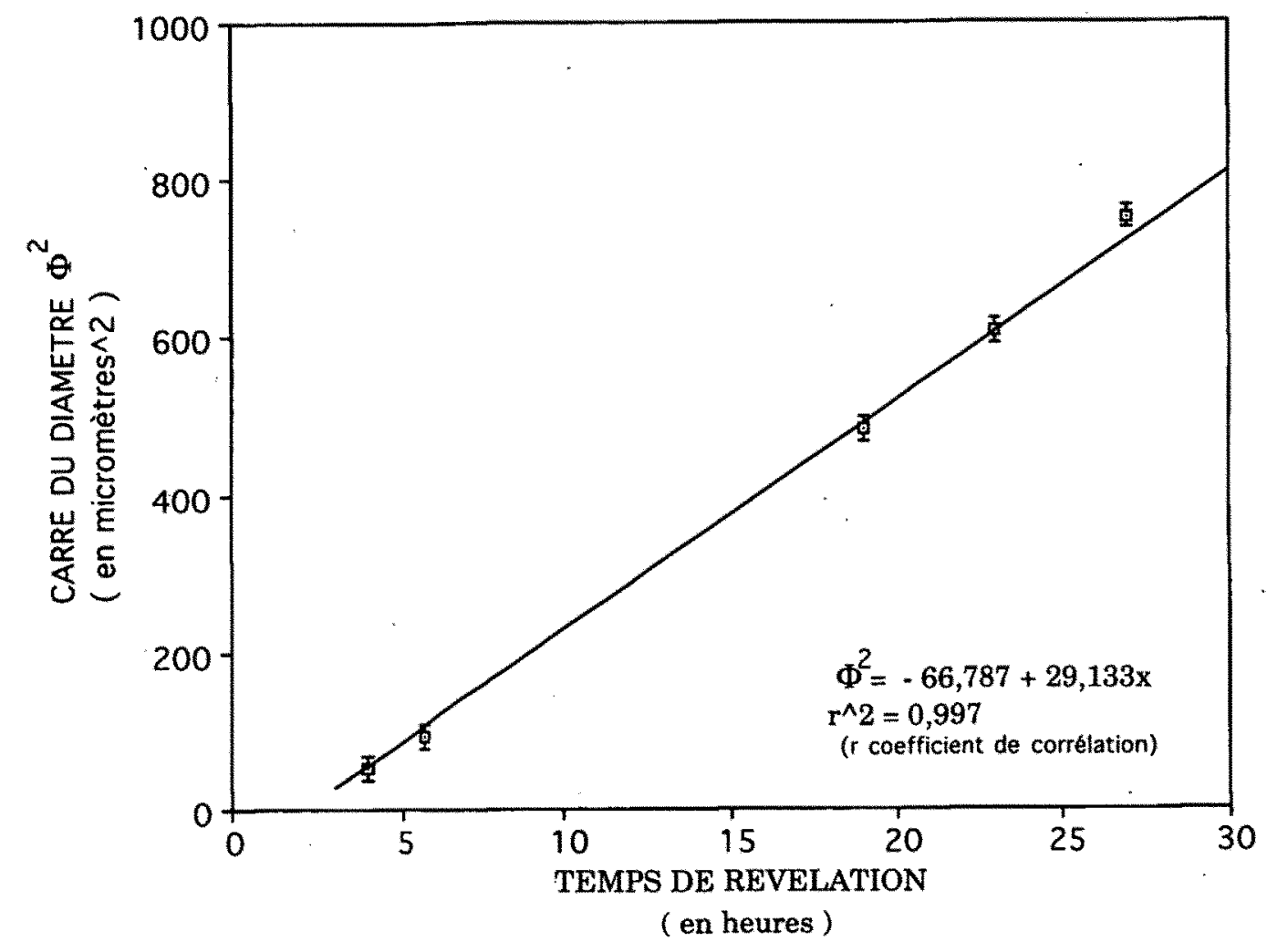

Fig. 5 - Valeurs des diamètres au carré des traces de protons pour l'énergie 351 keV en fonction du temps (incidence $=0^{\circ}$ ).

Values of the proton track square diameters for $351 \mathrm{keV}$ energy as a function of time.

Les valeurs de $a$ et $b$ trouvées nous permettent de calculer dans un premier temps les valeurs des parcours révélables $L$, puis celles des profondeurs des calottes $P_{C}$ et des temps $t_{L}$ associés. Ces résultats sont exprimés dans le tableau II. A partir de ce tableau, nous pouvons tracer le graphique du parcours en fonction de l'énergie (Fig. 8, parcours expérimental I). 
MESURE DU PARCOURS DE PROTONS DANS UN POLYMĖRE DÉTECTEUR

TABLEAU I

Coefficients $a$ et $b$ de régression linéaire et coefficient de corrélation $r$ pour les différentes énergies

Linear regression coefficients $a$ and $b$

and correlation coefficients $\boldsymbol{r}$ for the different energies

\begin{tabular}{|cccc|}
\hline $\begin{array}{c}\text { Energie des protons } \\
\text { (en keV) }\end{array}$ & $\begin{array}{c}a \\
\mathbf{m}^{2} \cdot \mathbf{h}^{-1}\end{array}$ & $\boldsymbol{u m}^{2}$ & $\begin{array}{c}\mathbf{r}^{2} \\
\left(\boldsymbol{r}=\text { coeff. }^{2}\right.\end{array}$ \\
\hline 238 & 16,803 & $-32,839$ & 1,000 \\
\hline 351 & 29,133 & $-66,787$ & 0,997 \\
\hline 576 & 56,339 & $-305,680$ & 0,971 \\
\hline 899 & 76,897 & $-839,130$ & 0,986 \\
\hline 1414 & 87,961 & $-1790,00$ & 0,970 \\
\hline
\end{tabular}

\section{d) Commentaires}

Bien que toujours supérieures, nos valeurs expérimentales de parcours sont du même ordre de grandeur que celles données par le logiciel TRIM 91 [3]. Nous avons, par exemple, une bonne concordance pour les basses énergies $(238,351,576 \mathrm{keV})$. Remarquons aussi qu'il y a bien corrélation entre la profondeur de la calotte et l'augmentation en énergie de la particule. Pour pousser plus en avant, nous voulons vérifier si la profondeur des calottes obtenue par le calcul concorde avec la réalité. Pour cela, nous avons utilisé la technique de microprofilométrie. Sur les détecteurs utilisés précédemment, nous allons mesurer les profondeurs $P_{\mathrm{C}}$ des calottes sphériques. Nous pourrons, de plus, recalculer $L$ en utilisant la relation suivante déduite des relations (1) et (2) :

$$
L=\frac{P_{\mathrm{C}}}{2}-\frac{b}{8 \cdot P_{\mathrm{C}}}
$$

\section{Mesure de la profondeur des calottes sphériques par microprofilométrie à palpeur tactile}

\subsection{Principe}

Ces mesures ont été réalisées à l'aide d'un profilomètre à palpeur tactile $[1,5]$, le capteur utilisant un stylet à pointe de diamant de rayon de courbure d'environ $2 \mu \mathrm{m}$, qui parcourt la surface à analyser selon plusieurs lignes voisines et parallèles. La résolution théorique de la mesure selon $Z$ (profondeur) est de l'ordre de $0,1 \mu \mathrm{m}$. 
TABLEAU II

Résultats des parcours $L$, des temps $t_{L}$ et des profondeurs $P_{C}$ pour chaque énergie

Results of ranges $L$, times $t_{L}$ and depths $\boldsymbol{P}_{C}$ for each energy

\begin{tabular}{|c|c|c|c|c|}
\hline \multicolumn{5}{|c|}{$\begin{array}{ccc}\text { Energie } & \begin{array}{c}E(\mu \mathrm{m}) \\
(\mathrm{keV})\end{array} & \mathrm{E}(\mu \mathrm{m}) \\
\mathrm{expérimental} & \mathrm{TRIM91} \\
\end{array}$} \\
\hline 238 & $\begin{array}{c}4,2 \\
( \pm 1,0)^{*}\end{array}$ & 2,7 & $\begin{array}{r}1,6 \\
( \pm 0,4)\end{array}$ & $\begin{array}{c}1,0 \\
( \pm 0,2)\end{array}$ \\
\hline 351 & $\begin{array}{r}5,4 \\
( \pm 0,9)\end{array}$ & 4,2 & $\begin{array}{r}1,9 \\
( \pm 0,2)\end{array}$ & $\begin{array}{c}1,9 \\
( \pm 0,2)\end{array}$ \\
\hline 576 & $\begin{array}{c}12,2 \\
( \pm 1,0)\end{array}$ & 8,4 & $\begin{array}{r}4,4 \\
( \pm 0,4)\end{array}$ & $\begin{array}{r}3,6 \\
( \pm 0,2)\end{array}$ \\
\hline 899 & $\begin{array}{c}23,5 \\
( \pm 0,7)\end{array}$ & $\begin{array}{l}16,3 \\
-\end{array}$ & $\begin{array}{r}9,6 \\
( \pm 0,3)\end{array}$ & $\begin{array}{r}5,0 \\
( \pm 0,2)\end{array}$ \\
\hline 1414 & $\begin{array}{c}42,0 \\
( \pm 1,4)\end{array}$ & 33,0 & $\begin{array}{c}18,8 \\
( \pm 0,3)\end{array}$ & $\begin{array}{c}5,4 \\
( \pm 0,4)\end{array}$ \\
\hline
\end{tabular}

* Entre parenthèses les écarts sur les mesures.

\subsection{Expériences et résultats}

Nous avons utilisé les échantillons qui nous ont déjà servi pour établir la courbe parcours/énergie (parcours expérimental I) de la figure 8 . Cette manipulation a pour but de confirmer notre modèle : pour des traces de protons de même énergie et d'incidence normale surrévélées, nous devons observer des calottes sphériques de profondeur indépendante du temps de développement. Les résultats sont présentés dans le tableau III. A partir de ce tableau, nous pouvons tracer le graphe du parcours en fonction de l'énergie (Fig. 8, Parcours expérimental II). La figure 6 montre une vue en perspective d'une trace surrévélée et visualise plusieurs de ses profils. La figure 7 compare le profil expérimental médian et celui théorique de cette trace surrévélée. Le rayon $R$ théorique (de la sphère limite de révélation) est déduit de la formule suivante :

$$
R=V_{\mathrm{B}} \cdot\left(t-t_{L}\right)=\frac{a}{8 \cdot P_{\mathrm{C}}} \cdot t+P_{\mathrm{C}}-L
$$


MESURE DU PARCOURS DE PROTONS DANS UN POLYMÈRE DÉTECTEUR

TABLEAU III

Résultats des mesures microprofilométriques Microprofilometric measurements

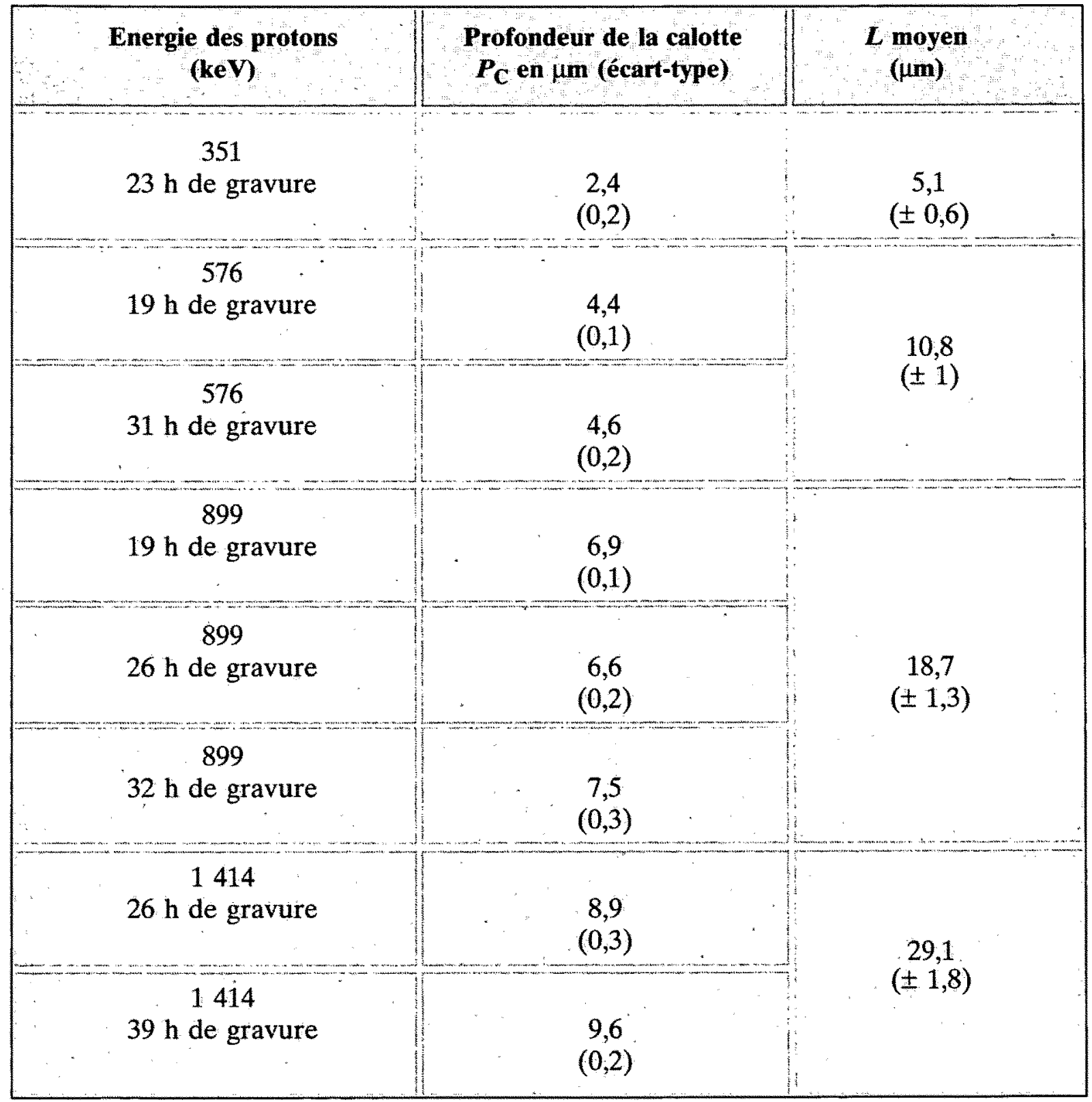

* Pour $L$ sont figurés entre parenthèses les écarts sur les mesures.

\subsection{Discussion}

Les mesures microprofilométriques confirment l'obtention de calottes sphériques lors de la phase de surdéveloppement. En effet, le profil théorique d'une calotte sphérique se superpose, aux incertitudes expérimentales près, à celui obtenu expérimentalement. De plus, nous pouvons considérer les profondeurs des calottes constantes au cours du temps. Cependant, elles sont toujours supérieures à celles que nous pensions obtenir avec $V_{\mathrm{B}}$ égale à $1,92 \mu \mathrm{m} \mathrm{h}^{-1}$. Les différences relevées peuvent être imputées aux variations de température même 
ENERGIE : $899 \mathrm{KeV}$

INCIDENCE : $0^{\circ}$

DUREE DE LA REVELATION : 32 HEURES
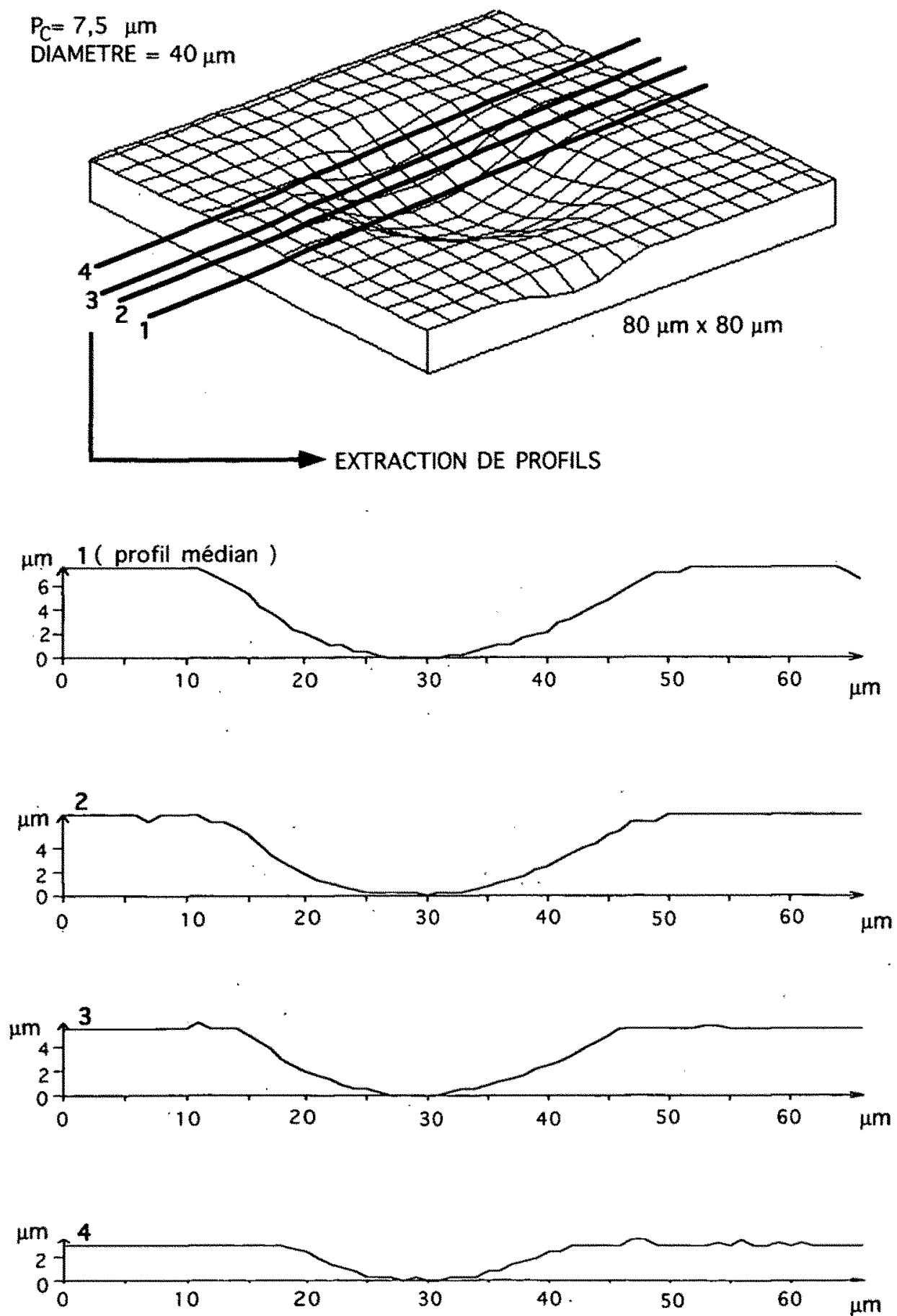

Fig. 6 - Vue et profils d'une trace surrévélée (énergie : $899 \mathrm{keV}$, incidence : $0^{\circ}$, durée de la révélation : $32 \mathrm{~h}$ ).

View and profiles of an overetched track. 
MESURE DU PARCOURS DE PROTONS DANS UN POLYMĖRE DÉTECTEUR

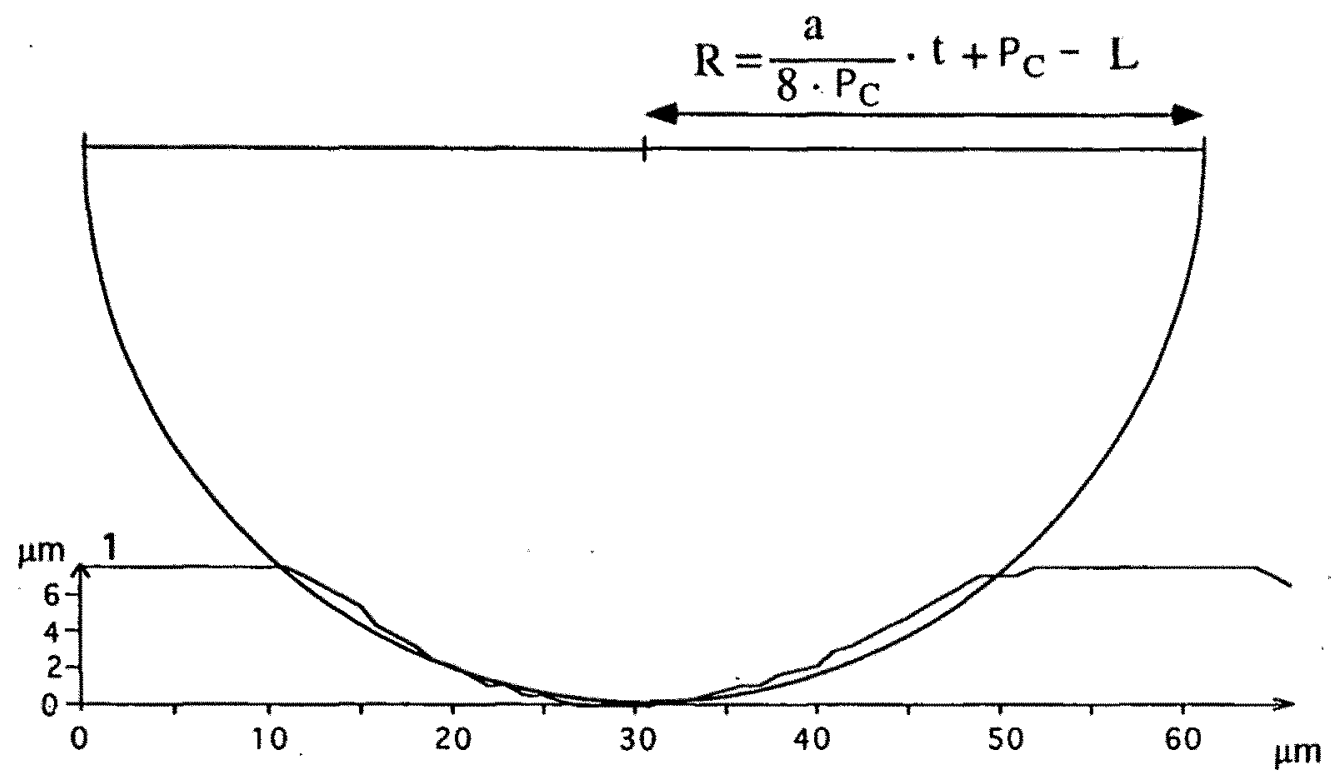

Dans ce cas $P_{\mathrm{C}}=7,5 \mu \mathrm{m} ; t=32 \mathrm{~h} ; L=18,7 \mu \mathrm{m} ; a=78 \mu \mathrm{m}^{2} \cdot \mathrm{h}^{-1} ; \mathrm{d}$ 'où $R=30,4 \mu \mathrm{m}$

Fig. 7 - Comparaison du profil expérimental médian et du profil théorique de la trace visualisée figure 9.

Experimental median profile vs theoretical profile of the track visualized figure 9.

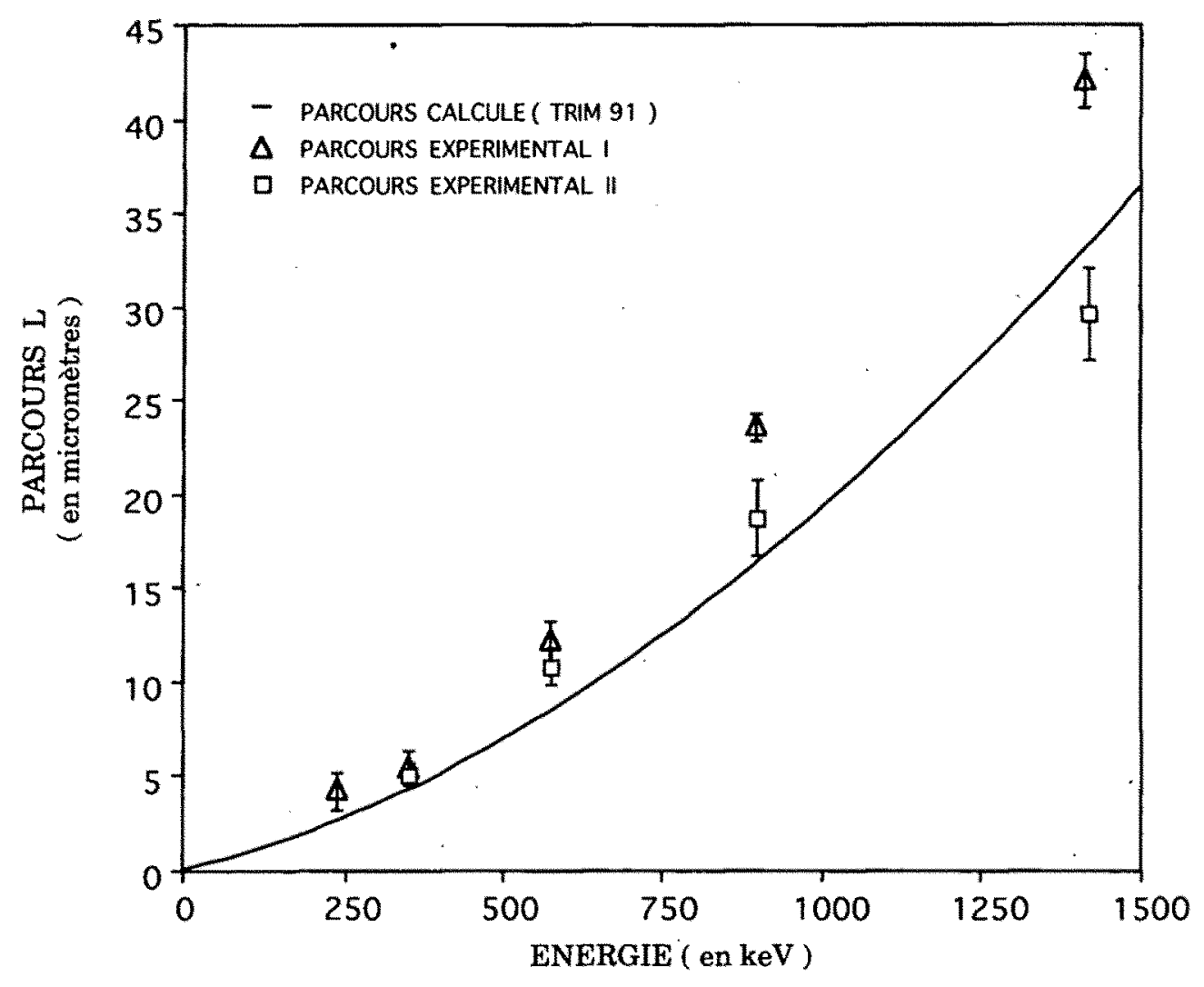

Fig. 8 - Parcours expérimentaux et théoriques des protons à incidence normale en fonction de l'énergie.

Experimental and theoretical ranges of normal incident protons vs energy. 
minimes sur des temps très longs. En effet, la vitesse $V_{\mathrm{B}}$ dépend de la température suivant une loi exponentielle [9]. Ces résultats sont, cependant, satisfaisants dans la mesure où les parcours $L$ calculés avec les valeurs expérimentales de $P_{\mathrm{C}}$ sont encore plus proches des valeurs calculées par TRIM 91.

La figure 8 regroupe tous les résultats de parcours :

- le parcours calculé donné par TRIM 91,

- le parcours expérimental I,

- le parcours expérimental II (obtenu en tenant compte des valeurs expérimentales de $P_{\mathrm{C}}$.

\section{Conclusion}

Grâce aux diverses techniques utilisées, nous avons pu valider notre analyse de la phase de surdéveloppement : profondeur des calottes sphériques constante, croissance linéaire du carré du diamètre en fonction du temps.

Nous avons remarqué que les valeurs expérimentales de parcours sont du même ordre de grandeur que celles calculées par le logiciel TRIM 91. Ces valeurs obtenues sont intéressantes, car à notre connaissance il n'existe pas de méthode expérimentale de mesure de parcours de particules chargées dans un solide. Il faut noter, cependant, que d'autres expériences devront être à nouveau effectuées (avec un échantillonnage plus important en énergie pour augmenter le nombre de points). Cela d'une part pour.confirmer ces résultats, et d'autre part pour qu'ils soient statistiquement satisfaisants (reproductibilité et précision). Les différences relevées ne remettent pas en cause la validité du logiciel TRIM 91.

Il sera nécessaire aussi de prendre en compte et, si possible, d'atténuer les variations de $V_{\mathrm{B}}$. Rappelons que ces dernières sont causées par d'éventuelles variations de la température au cours de la gravure. De la stabilité de ce paramètre thermodynamique dépendent les variations de $V_{\mathrm{B}}$. Avec notre méthode, des variations de température mêmes minimes faussent les résultats du parcours. La conséquence de ces variations indésirables apparaît à travers les différences entre les parcours expérimentaux I et. II, car le second prend en compte les variations de $V_{\mathrm{B}}$.

La technique que nous avons proposée pour les protons peut être transposée au cas des deutons, des tritons, des hélions et des particules alpha pour tout type de DSTN. Pour des ions plus lourds, la trace latente possédant des dimensions radiales de l'ordre de grandeur des parcours, il faudrait, en toute rigueur, tenir compte des variations radiales de la vitesse latérale de développement dans le corps de la trace. Dans ce cas, notre modèle n'est plus approprié, parce qu'il utilise une vitesse $V_{\mathrm{B}}$ constante et ne tient pas compte de ces variations radiales dans le corps de la trace latente. 
Cette étude constitue une mise au point. La méthode proposée permet d'obtenir la relation expérimentale parcours/énergie ainsi que l'expression de la vitesse $V_{\mathrm{T}}$ d'attaque chimique le long de la trace latente. Ces deux dernières données sont indispensables à l'expérimentateur quand celui-ci effectue un travail de dosimétrie ou de cartographie par DSTN.

\section{RÉFÉRENCES}

[1] ASSOUL M. - Analyse de la topographie des surfaces quelconques. Thèse, Besançon, 1991.

[2] BETH M., ENGE W., SERMUND G. - Some new aspects on etching of CR 39 plastic detector. Nucl. Tracks Radiat. Meas., 1986, 12 (1/6), 91-95.

[3] BIERSACK J.P.; ZIEGLER J.F. - Logiciel "TRIM 91". New York : Pergamon Press, 1992.

[4] CARTWRIGHT B.G., SHIRK E.K., PRICE P.B. - A nuclear-track recording polymer of unique sensitivity and resolution. Nucl. Instrum. Methods, 1978, $153(2 / 3)$, 457-460.

[5] CHUARD M., RONDOT A.C., MIGNOT J. - On the use of a modular system of microtopographical surface measurement. Wear, 1984, 96, 31-44.

[6] FEWS A.P. - A study of alpha particle tracks in CR 39 plastic. Thesis, University of Bristol, June 1992.

[7] FEWS A.P., HENSHAW D.L. - High resolution alpha spectroscopy using CR 39 plastic track detector. Nucl. Instrum. Methods, 1982, 197, 517-529.

[8] FLEISCHER R.L., PRICE P.B., WALKER R.M. - Nuclear tracks in solids, principles and methods. Los Angeles : University of California Press, 1975.

[9] FROMM M., MEMBREY F., CHAMBAUDET A., SAOULI R., EL-RAHAMANY A. - A study of CR 39 bulk etch properties under various temperature and concentration conditions to modelize the dissolution rate. Nucl. Tracks Radiat. Meas, 1991, 19 (1/4), 169-170.

[10] FROMM M. - Evaluation des potentialités d'un polymère isotrope (CR 39) en tant que détecteur de traces nucléaires pour la dosimétrie neutron et la microcartographie alpha. Thèse, Besançon, 1990.

[11] FUJII M., NISHIMURA J. - Generalized etch-pit equations and their applications to analyses of tracks in CR 39, with depth-dependent etching properties. Nucl. Tracks Radiat. Meas, 1986, $11(1 / 2), 25-33$.

[12] MEMBREY F., CHAMBAUDET A., FROMM M., SAOULI R. - Track etching model for normal incident heavy ion recording in isotropic dielectric detectors. Nucl. Tracks Radiat. Meas, 1990, 17 (3), 183-190.

[13] SOMOGYI G., SZALAY S.A. - Track-diameter kinetics in dielectric track detectors. Nucl. Instrum. Methods, 1973, 109, 211-232.

[14] VAREILLE J.C. - Répartition des dommages créés autour de la trajectoire d'un ion dans le triacétate de cellulose. Thèse, Limoges, 1982. 